\title{
A virtual human agent model with behaviour based on feeling exhaustion
}

\author{
Jan Treur
}

Published online: 8 June 2010

(c) The Author(s) 2010. This article is published with open access at Springerlink.com

\begin{abstract}
A computational agent model for monitoring and control of a virtual human agent's resources and exhaustion is presented. It models a physically grounded intelligent decision making process within the agent model for physical effort to be spent. Simulation results are discussed, and a formal analysis is presented on conditions under which the agent model functions properly, for example, such that it can be used to avoid running out of resources. The model is related to a model for monitoring or simulating a person's heart rate. Finally some validation experiments are briefly discussed.
\end{abstract}

Keywords Agent model · Exhaustion · Fatigue - Virtual · Energy use regulation $\cdot$ Simulation $\cdot$ Mathematical analysis

\section{Introduction}

To generate intelligent agent behaviour, it is more and more recognized that in addition to the brain, the body often plays a crucial role as well, and thus contributes to the intelligence. Some authors argue that also the design of artificial intelligent systems could gain benefit of such analyses by incorporating relevant physiological aspects in models developed; e.g., [3, 4, 6, 18]. An example is the intelligence with which a person manages exhaustion (or fatigue). In the

A preliminary, shorter version of this paper was included as a full paper in the IEA/AIE'09 conference.

J. Treur $(\bowtie)$

Department of Artificial Intelligence, Vrije Universiteit Amsterdam, De Boelelaan 1081, 1081 HV, Amsterdam,

The Netherlands

e-mail: treur@cs.vu.nl

url: http://www.cs.vu.nl/ treur case the body would only give a signal when complete exhaustion occurs, no intelligent management would be possible. Fortunately, by gradually getting a feeling of becoming fatigued, more information is available before a total breakdown occurs. In this paper a computational model and analysis is made of the role that this physiological aspect (as described in more detail in the literature on physical exercise and sport) plays in monitoring and intelligent control of resources.

For certain types of artificial or virtual human agents, monitoring and control of their own resources may be of importance, for example, for human-like characters in a virtual reality context. When such a virtual human agent is equipped with the capability to get a feeling of becoming fatigued, it may show a more realistic intelligent behaviour. This behaviour can be based on a context-sensitive type of decision making to manage limitations of resources, incorporating the intelligence related to aspects of the body. Another application area is formed by ambient intelligence used in physical exercise and sport: devices that monitor human functioning, are able to analyse this functioning, and respond accordingly. When more sophisticated agent models are used, more advanced ambient intelligent agent applications can be developed.

In this paper literature on physical exercise and sport (e.g., [19]) is taken as a point of departure. One of the issues addressed is how the generated effort is controlled and what is the role of feeling fatigue in this process; e.g., [5, 7-15, 17]. The interplay of mind and body in this process is considered a crucial factor. The classical perspective on fatigue is based on the assumption that fatigue occurs either when the muscles run out of resources, or they are in a sense poisoned by waste material produced; e.g., [10-12]. Resources may involve oxygen, glycogen (which fuels the muscles), or ATP (adenosine triphosphate, the molecule that muscles use 
to store energy) that are lacking. Waste material concerns by-products of exercises, such as lactic acid. In this perspective the body reaches some states in which its functioning is disturbed so that only limited effort is possible, and this co-occurs with (or is expressed by) feeling fatigue. Noakes and his colleagues (e.g., $[14,15])$ emphasize the notion of homeostasis: the property of a system (for example, a living organism), to regulate its internal environment in such a way that stable, more or less constant, conditions are maintained. They claim that:

- Athletes can become fatigued even when they have enough oxygen left in their blood to keep going for some time.

- Fatigue is not always accompanied by the build-up of lactic acid.

- Glycogen and ATP stores are not always polished off during endurance events.

According to them the body is rarely allowed to reach a 'catastrophic' state where it would run out of all such essential reserves. In this view the mind keeps the body in physical conditions that are better prepared for expected or possible future efforts. A cognitive or neural decision making mechanism is assumed that incorporates information on the extent of exhaustion of the agent's body. Noakes et al. formulate the following assumptions: ${ }^{1}$

- There is never complete muscle recruitment during voluntary exercise in humans.

- Homeostasis is maintained in all forms of exercise.

- Fatigue is a symptom that is linked in some way to the maintenance of homeostasis. Thus the greater the effort required to maintain homeostasis, the greater the symptoms of fatigue. Indeed the perception of fatigue during exercise rises as a linear function of exercise duration suggesting that, already at the onset of exercise, the brain has calculated for how long it will allow that exercise about to continue and that it is the achievement of a specific level of fatigue sensations that causes the voluntary termination of exercise.

- The protection of homeostasis during exercise is hardwired in the brain but can be modified by a number of interventions including training, recent exercise and acute metabolic changes such as exposure to low blood glucose or oxygen concentrations.

According to this perspective it is not the body that limits the mind, but the other way around: the mind limits the body, thus keeping the body in physical conditions that are better prepared for expected or possible future efforts. Here a decision making mechanism is at work that, in addition to interests in the present, also involves interests in the future. This

\footnotetext{
${ }^{1}$ See, e.g., http://www.mrc.co.za/exercise/projects9.htm.
}

is an example of a type of decision making where choice options for different points in time are compared, called intertemporal decision making.

To obtain a more specific context to analyse this phenomenon, the case study in this paper will be on physiological effort in cycling, and in particular on the control of effort to enable management of resources. The power provided by a cyclist is used to work against resistance of three types: mechanical (the bike's mechanisms), air (the wind, including the wind encountered due to the cyclist's own speed), and gravitational resistance (for climbing). When speed is not very low, and the road is flat, air resistance is the main factor. Cyclists often cooperate to reduce air resistance: riding behind each other.

In Sect. 2 the computational agent model for monitoring and management of resources is presented and formalised. Section 3 discusses simulation results for the domain of cycling, which is used as a case study. In Sect. 4 a formal analysis is presented, which identifies the conditions and parameter values for which the model will function properly. Section 5 briefly analyses how the agent model can be related to a person's heart rate, which is sometimes used as monitor information in sports. Section 6 is a discussion.

\section{The computational agent model}

This section describes the agent model for monitoring and control of resources. A central concept used is power that is generated. The basic idea behind the model is that it is easier to monitor the generated power at any time point, than the store of resources left. When based on monitor data on the generated power, the brain performs some form of accumulation or integration, then this can be used as a faithful indicator for the resources used. Within the literature on exercise and sports the notion of critical power CP plays an important role. This is the maximal level of power that can be generated and sustained over longer periods without becoming exhausted, assuming no prior exercising. It is an asymptote of the hyperbolic power-duration curve defined by $(G P-C P) . t=W^{\prime}$ that (as shown in various experiments) models the relationship between a constantly generated power $G P$ (above the critical power $C P$ ) and the time $t$ that this can be sustained; e.g., [7-12, 17]. Here $W^{\prime}$ is the total amount of work that can be spent above the critical power (the available stored resources). Often it is (implicitly or explicitly) assumed that this critical power $C P$ is a constant, that is not affected by prior exercising, and is a capacity to provide (sustainable) power based on aerobic processes. Power generated above this critical power is assumed to be based on (nonsustainable) anaerobic processes, that exploit an available fixed reservoir of stored basic resources $B R$, which is one of the parameters of the hyperbolic 
Table 1 Most important variables of the model

\begin{tabular}{ll}
\hline Notation & Description \\
\hline$G P$ & Generated power \\
$P P$ & Provided power \\
$R P$ & Recovery power \\
$E P$ & Extracted power \\
$D C P$ & Dynamical critical power \\
$B C P$ & Basic critical power \\
$L C P$ & Lowest critical power \\
$D M P$ & Dynamic maximal power \\
$B M P$ & Basic maximal power \\
$A C P$ & Actual cycling power \\
$C E F$ & Cycling efficiency factor \\
$A R C$ & Air resistance coefficient \\
\hline
\end{tabular}

power-duration curve (often indicated by $W^{\prime}$ ). Experiments show that after highly intensive prior exercising leading to exhaustion of the basic resources $B R$, for example, power at $90 \%$ of the critical power $C P$ cannot be sustained; e.g., [5]. Therefore a main assumption made for the model developed below is that a basic critical power BCP (applicable when no prior exercising took place) can be distinguished from a dynamic critical power DCP (applicable when prior exercising has taken place). The most basic assumptions behind the model are:

- The dynamic critical power indicates the level of (sustainable) power that can be generated for which the level of fatigue remains the same.

- If a level of power is generated above the dynamic critical power, then fatigue will increase which makes that less sustainable power can be generated.

- If a level of power is generated below the dynamic critical power, then fatigue will decrease which makes that more sustainable power can be generated.

- Lower dynamic critical power DCP reflects more fatigue, and the maximal dynamic critical power DCP (which is the basic critical power BCP) means no fatigue, occurring in a state without prior exercising.

Note that in Table 1 an overview is given of the symbols used. The upper bound for the dynamic critical power is the basic critical power $B C P$ and a lower bound is lowest critical power LCP.

Part of the dynamics of the model concerns how this dynamic critical power is affected by the effort spent above the critical power in the preceeding time interval (i.e., the extent to which the basic resources $B R$ were already used), and how recovery can take place when the effort spent is below the critical power. Because the dynamic critical power has a direct relationship with the history of effort spent, it can be compared to the feeling of becoming fatigued, which in-

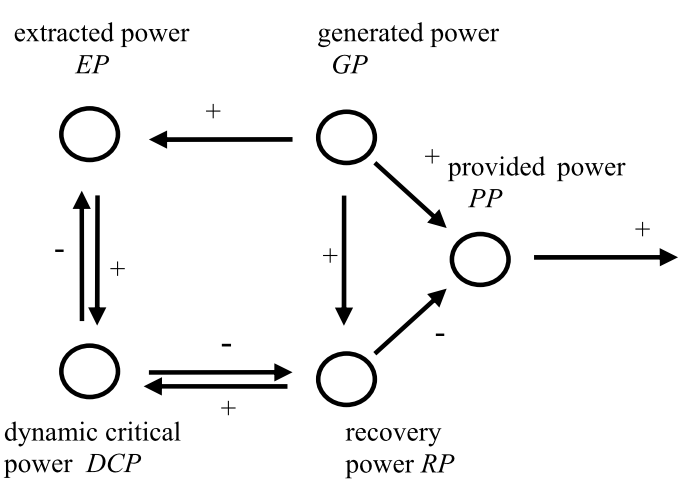

Fig. 1 Overview of the variables and their dependencies

dicates the extent of exhaustion, or the state of the (remaining) resources. In this way the dynamic critical power can be taken as a monitoring instrument to maintain homeostasis. Based on this indicator, decisions can be made on generated power. A possible decision model, for example, takes care that the dynamic critical power always is kept above a certain lower bound. When it is assumed that the dynamic critical power relates to fatigue (the lower the dynamic critical power, the more fatigue), this means that fatigue is kept limited. To obtain a formal model, the concepts used are formalised by numerical variables. Power is the amount of energy spent per time unit (expressed in Watt). Different types of power are distinguished; see Fig. 1 for the global effects they have on each other. Note that here the dynamic critical power $D C P$ has a temporal relation to the other nodes, whereas the others have state relations; below more specific formulae are discussed.

\section{Generated power and dynamic critical power $\rightarrow$ extracted power}

The generated power is the power level chosen by the person. If this is more than can be produced in a direct manner (by the aerobic system), part of this power is extracted from the (longer term) resources (the anaerobic system). When generated power is above the dynamic critical power, then the difference is extracted from the resources, indicated by:

$E P(t)=\operatorname{Pos}(G P(t)-D C P(t))$

where the operator Pos for the positive part is defined by $\operatorname{Pos}(x)=(x+|x|) / 2$, or, alternatively: $\operatorname{Pos}(x)=x$ if $x \geq 0$ and 0 else.

Generated power and dynamic critical power $\rightarrow$ recovery power

When the generated power is lower than the dynamic critical power, this means that not all available endurable resources are needed. Therefore it is assumed that a fraction of the generated power is contributed as recovery power. It is assumed that this recovery power is proportional to the difference 
between generated power and dynamic critical power, and also proportional to the difference between dynamic critical power and basic critical power. The proportion factor is $\beta$

$R P(t)=\beta G P(t) \operatorname{Pos}(D C P(t)-G P(t)) \frac{B C P-D C P(t)}{B C P}$

Extracted power and recovery power $\rightarrow$ dynamic critical power

Extracted power decreases the dynamic critical power. Recovery power increases the dynamic critical power, bounded upward by the basic critical power $B C P$. The adjustment of the dynamic critical power after a time interval from $t$ to $t+\Delta t$ is assumed proportional to the recovery power (factor $\gamma_{1}$ ), respectively the extracted power (factor $\gamma_{2}$ )

$D C P(t+\Delta t)=D C P(t)+\left(\gamma_{1} R P(t)-\gamma_{2} E P(t)\right) \Delta t$

The continuous model can be described by a differential equation:

$$
\begin{aligned}
\frac{d D C P(t)}{d t} \\
=\gamma_{1} R P(t)-\gamma_{2} E P(t) \\
=\gamma_{1} \beta G P(t) \operatorname{Pos}(D C P(t)-G P(t)) \frac{B C P-D C P(t)}{B C P} \\
\quad-\gamma_{2} \operatorname{Pos}(G P(t)-D C P(t))
\end{aligned}
$$

Recovery power and generated power $\rightarrow$ provided power The provided power is the difference between generated power and recovery power

$$
P P(t)=G P(t)-R P(t)
$$

\section{Dynamic critical power $\rightarrow$ dynamic maximal power}

The notion of maximal power models a limitation on the choice of the generated power. An assumption in the model is $^{2}$ : the higher dynamic critical power, the higher the dynamic maximal power. Extracted power can only be positive as long as the dynamic critical power is above its minimum value $L C P$. The maximum power possible $D M P$ is assumed to be the dynamic critical power plus a constant $C$, as long as $D C P>L C P$ (no complete exhaustion), and equal to $L C P$ when $D C P=L C P$ (complete exhaustion).

$$
\begin{aligned}
& D M P(t)=C+D C P(t) \quad \text { when } D C P>L C P \\
& D M P(t)=L C P(t) \quad \text { when } D C P=L C P \\
& B M P=C+B C P
\end{aligned}
$$

Here $B M P$ is the basic maximal power (when no exhaustion is present). Note that before reaching complete exhaustion,

\footnotetext{
${ }^{2}$ An alternative assumption could be that the maximal power is constant.
}

maximal power is substantially above the dynamic critical power, but upon reaching complete exhaustion, the maximal power drops to the level of the critical power, in accordance with the experiments reported, for example, in [4]. The agent model as described provides possibilities to make decisions based on the dynamic critical power as an indicator. When it is assumed that the dynamic critical power relates to the feeling of fatigue, then the idea is that the dynamical critical power indicator is available to the (virtual) human by experiencing this feeling.

\section{An example simulation for the cycling case}

In this section an example simulation for the cycling case is discussed. First a computational model for this case is introduced, as an extension to the more general model described above. Next, the example simulation is discussed and evaluated.

\subsection{The cycling case}

First a model for a cycling case study is described; see Fig. 2. In this case study the provided power is used to move a bike with a certain speed, depending on the resistance. Mechanical resistance can be taken into account in a cycling efficiency factor, for the process of generating actual power to move the bike. Further resistance is mainly based on air resistance, and if the road is ascending or descending on gravitation resistance. Given these resistances, velocities can be determined, and from them distances. For the sake of simplicity no gravitation resistance is considered; air resistance depends on a parameter called air resistance coefficient.

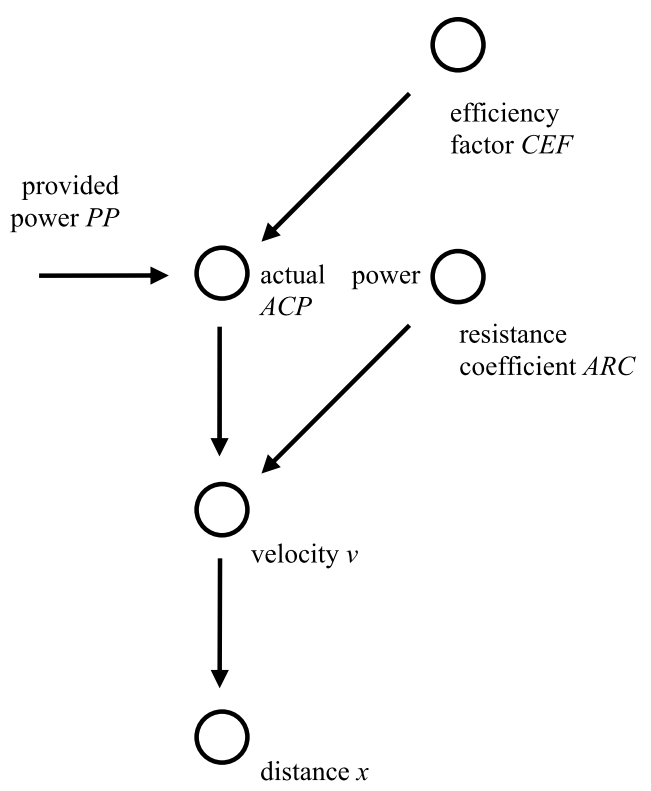

Fig. 2 Overview of the extension of the model for the cycling case 
To obtain a formal model, numerical variables are used: cycling efficiency factor $C E F$, air resistance coefficient $A R C$, actual cycling power $A C P$, and velocity $v$. The actual cycling power is the cycling efficiency factor times the provided power: $A C P(t)=C E F * P P(t)$. It is assumed that power exerted for movement is used to work against air resistance. For air resistance it is assumed that it is a force proportional to the square of velocity, with resistance coefficient $A R C$. Actual cycling power is the work performed per time unit, which is equal to this resistance force times the distance covered divided by the time (which is the velocity); therefore $A C P(t)=A R C * v(t)^{3}$ or:

$v(t)=\sqrt[3]{\frac{A C P(t)}{A R C}}$

\subsection{The example simulation}

Based on the model described in Sect. 2 and the cycling model described above, a number of simulation experiments have been performed, using common numerical simulation
Fig. 3 Example simulation trace for the cycling case
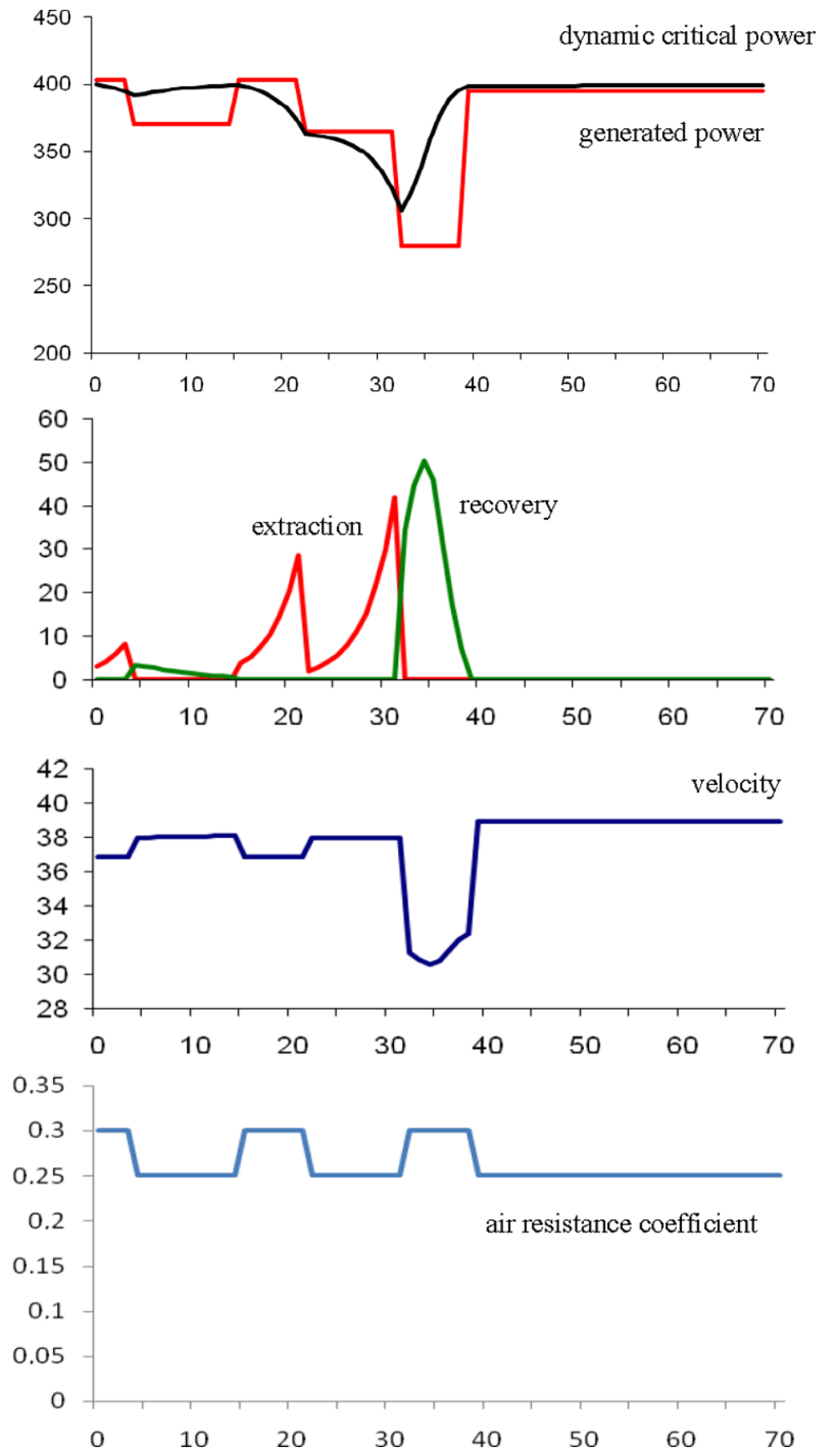
tools such as Microsoft Excel or MathLab. In Fig. 3 results are shown of one of them, with time scale displayed in minutes; the step size $\Delta t$ was taken one minute. The fixed parameter settings are:

$\beta=0.02$

$\gamma_{1}=\gamma_{2}=0.4$

$\rho=0.8$

$B C P=400$

$L C P=300$

$C=100$

The story goes as follows. First the cyclist generates power a bit above (403) the dynamic critical power (initially 400), riding alone (air resistance coefficient 0.3 ). As this effort higher than the dynamic critical power cannot be sustained, the dynamic critical power slightly decreases: more fatigue. Then she joins a group of cyclists that passes by, and hence has less air resistance (coefficient 0.25 ). The generated power is now lower than the dynamic critical power, while the speed is higher. Therefore some recovery takes place. After a while in the group she is persuaded that it is her turn to take the front position. Now she has higher air resistance again, upon which she generates higher power (but lower speed), which is not sustainable; this brings the dynamic critical power down to near 350. After some time she leaves the front position to somebody else, and while still being in the group she has less air resistance. However, as the dynamic critical power was decreased, the generated power now is still above the dynamic critical power; the dynamic critical power continues to decrease and approaches 300: she cannot maintain the speed of this group after her effort at the front. She decides to leave the group and take some recovery time, riding on her own, with much lower speed, with more air resistance. The dynamic critical power increases; when another group passes by, she joins this group, and has lower air resistance again. Now the generated power stays slightly under the dynamic critical power, although this group has a higher speed. A state occurs with (almost) constant dynamic critical power.

Note that after 30 minutes a critical situation occurred. Taking the front position in the (first) group took so much of the resources that after leaving the front position, still the generated power to stay within the group was higher than the dynamic critical power. Therefore the cyclist could not recover and instead had to extract more and more from the resources, bringing the dynamic critical power further down. If no decision would have been made to decrease the generated power, within a very short time the decreasing trend of the dynamic critical power would have led to the lower bound $L C P$ of the dynamic critical power, after which the maximal generated power would drop to the level of $L C P$.
The decisions of the cyclist have been modelled by the generated power levels over time, set by hand (see the lowest graph in Fig. 3). Note that in the second picture in Fig. 2, the areas under the extraction graph and the recovery graph are more or less the same, which means that the dynamic critical power after 40 minutes is almost equal to the one at the start, which also can be seen in the first graph.

\subsection{Evaluation of characteristic patterns}

A number of expected characteristic patterns were formulated initially. For each of them it is discussed in how far they are realised by the model.

Keeping the energy-related parameters of the organism between certain limits (homeostasis)

The model formalises the perspective that fatigue plays a role in a cognitive or neural mechanism that allows an agent to maintain homeostasis: keeping the energy-related parameters of the agent, in particular the resources that are present in the body, between certain limits. The model is based on monitoring of the generated power at any time point. It performs integration of these monitoring data over time and uses this to determine indicators for the power extracted from the resources, the recovery of the resources and the amount of resources left. The model indeed realises homeostasis under the assumption that the human makes the proper decisions about its generated power. Appropriate decisions about generated power can be based on certain indicators provided by the model: the stable power point (assuming that this correlates to feeling fatigue), the stable heart rate, or the heart rate itself. Decision criteria for generated power can be simple: keeping such indicators constant to achieve a steady state, or keeping the indicators between certain bounds, thus avoiding running out of resources.

The effect of differences in the resistance, for example, based on riding behind somebody else, in order to reduce air resistance

These effects are also realised in the model. In the example simulation it is shown how the cyclist provides a higher speed (for lower generated power) when joining a group.

Irregular effort is less efficient than regular effort (for reaching a distance in a time interval)

This has been shown by multiple simulations of the model. In particular, the fact that air resistance is not linear with speed makes that irregular effort is less economical than regular effort.

\section{Formal analysis}

A main question addressed in the formal analysis is whether the introduced agent model allows the person to monitor and 
control its resources in a proper manner. For some lemmas and proof sketches, see Appendix.

\section{Maintaining a steady state}

Endurance sporters often try to maintain what they call a steady state: a state in which the main parameters are kept constant. In particular, it can be analysed how $D C P(t)$ can be kept constant.

An analysis of this is summarised in the following theorem expressing how a steady state for $D C P(t)$ can be characterised.

Theorem 1 (Maintaining a steady state)

For any time point $t$, when $G P(t)>0$, the following are equivalent:

(i) $\frac{d D C P(t)}{d t}=0$ (dynamic critical power equilibrium)

(ii) Either $G P(t)=D C P(t)$ or $G P(t) \leq D C P(t)=B C P$

How the indicator relates to the resources

In Proposition 1 it is investigated under which conditions the dynamic critical power is a proper indicator for the basic resources. It turns out that this is the case under a certain condition on the parameters; the same condition implies that the resource level is a linear function of the dynamic critical power. Here $R(t)$ denotes the remaining resources at $t$ and $B R$ the (initial) basic resources.

Proposition 1 (Indicating basic resource levels)

The following are equivalent:

(i) The dynamic critical power is a proper indicator for basic resources:

$\forall t \quad[D C P(t)=B C P \Leftrightarrow R(t)=B R]$

(ii) $\gamma_{1} / \alpha_{1}=\gamma_{2} / \alpha_{2}$

(iii) For all time points $t_{1}$ and $t_{2}$ it holds:

$\alpha_{1}\left(D C P\left(t_{2}\right)-D C P\left(t_{1}\right)\right)=\gamma_{1}\left(R\left(t_{2}\right)-R\left(t_{1}\right)\right)$

(iv) The expression $\gamma_{1} R(t)-\alpha_{1} D C P(t)$ is invariant over time: for all time points $t_{1}$ and $t_{2}$ it holds:

$\gamma_{1} R\left(t_{2}\right)-\alpha_{1} D C P\left(t_{2}\right)=\gamma_{1} R\left(t_{1}\right)-\alpha_{1} D C P\left(t_{1}\right)$

(v) The dynamic critical power is a linear function of the resource level: for a given point $t_{0}$, for all time points $t$ it holds:

$\alpha_{1} D C P(t)=\gamma_{1} R(t)+\alpha_{1} D C P\left(t_{0}\right)-\gamma_{1} R\left(t_{0}\right)$

In Proposition 2 it is investigated under which conditions the dynamic critical power is a proper indicator for running out of resources. This is the case under a certain further condition on the parameters; this condition implies that the resource level is proportional to the dynamic critical power, as expressed in Theorem 2. Below it is assumed that at the initial time point $t_{0}$ the resources $R\left(t_{0}\right)$ are the basic resources $B R$ and the dynamic critical power $D C P\left(t_{0}\right)$ is the basic critical power $B C P$.

Proposition 2 (Indicating running out of resources) Suppose $\gamma_{1} / \alpha_{1}=\gamma_{2} / \alpha_{2}$ and let $\eta=B C P / B R$. Then the following are equivalent:

(i) The dynamic critical power is a proper indicator for running out of resources:

$\forall t \quad[D C P(t)=L C P \Leftrightarrow R(t)=0]$

(ii) At any point of time the dynamic critical power as an indicator faithfully (proportionally) reflects the resources left:

$(D C P(t)-L C P) / R(t) \quad$ is constant over time

(iii) $\gamma_{1} / \alpha_{1}=\gamma_{2} / \alpha_{2}=\eta$

Theorem 2 (Proper indicator for resources) In case $\gamma_{1} / \alpha_{1}=$ $\gamma_{2} / \alpha_{2}=\eta$, with $\eta=B C P / B R$, the following hold:

(a) The dynamic critical power is a proper general indicator for the resource level. More specifically, $D C P(t)$ $L C P$ is proportional to $R(t)$ over time with $\eta$ as factor:

$$
\forall t \quad D C P(t)=L C P+\eta R(t)
$$

(b) Suppose for some lower bound $L>L C P$ the basic power $D C P(t)$ is always kept above $L$ i.e., $\forall t$ $D C P(t) \geq L$, then the person will never run out of resources

(c) When the person has nonzero resources and $D C P(t)$ is kept constant, then the person will never run out of resources

In this theorem (a) and (b) immediately follow from Proposition 2. Concerning (c), when the person keeps $D C P(t)$ at a constant value above $L C P$, then (b) applies. Therefore the person can only run out of resources when $D C P(t)=L C P$, but as $D C P(t)$ is kept constant, then by Proposition 2 there already were no resources, which is not the case.

\section{Corollary}

In case $\gamma_{1} / \alpha_{1}=\gamma_{2} / \alpha_{2}=\eta$, with $\eta=B C P / B R$, the resource level $R(t)$ over time satisfies the following differential equation

$$
\begin{aligned}
& \frac{d R(t)}{d t} \\
& =\alpha_{1} \beta G P(t) \operatorname{Pos}(\eta R(t)+L C P-G P(t)) \frac{B R-R(t)}{B R} \\
& \quad-\alpha_{2} \operatorname{Pos}(G P(t)-\eta R(t)-L C P)
\end{aligned}
$$




\section{Relation to heart rate}

In exercising and sport practice sometimes not (only) the feeling of fatigue, but (also) the heart rate is used to monitor the extent of exhaustion; here often some estimations are used, assuming a linear relation of heart rate to generated power above the critical power point. For example, if it is assumed that the heart rate for generated power at the level of the critical power is a constant $B H R$, and the maximal heart rate is a constant $M H R$, then for cases where $G P(t)$ is at least $D C P(t)$ the heart rate can be estimated as:

$$
\begin{aligned}
H R(t)= & B H R+(M H R-B H R)(G P(t)-D C P(t)) \\
& /(D M P(t)-D C P(t)) \\
= & B H R+(M H R-B H R)(G P(t)-D C P(t)) / C \\
= & B H R+\gamma(G P(t)-D C P(t))
\end{aligned}
$$

with $\gamma=(M H R-B H R) / C$. From this relation it follows that

$\frac{d H R(t)}{d t}=\gamma \frac{d G P(t)}{d t}-\gamma \frac{d D C P(t)}{d t}$

Therefore in case of generated power in equilibrium (for example, constant), the following can be derived.

Theorem 3 (Heart rate as indicator)

(a) If $G P(t) \geq D C P(t)$ and it holds $\frac{d G P(t)}{d t}=0$ (generated power equilibrium) then the following are equivalent:

(i) $\frac{d H R(t)}{d t}=0$ (heart rate equilibrium)

(ii) $\frac{d D C P(t)}{d t}=0$ (critical power equilibrium)

(b) When both the generated power and heart rate are constant, then no running out of resources will occur

\section{On validation of the model}

The agent model may be applied as part of an intelligent ambient agent device interacting with humans in physical exercise or sport, or in other demanding circumstances. Supporting devices mostly concentrate on the sensoring and usually do not possess much intelligence to perform analysis of sensor data. Incorporating a dynamical model may provide a basis for more intelligent ambient agents in relation to such devices. It is in this direction that some initial validation of the model has taken place. First it is discussed how the model was used in projects of first year undergraduate students in AI, addressing the design and use of an ambient agent to guide cycling training. Next it is discussed how the presented model was integrated in a model for an operator's functional state in demanding tasks, and how this more complex model was validated.
6.1 Validation of the model by experiments in guiding cycling training

In the final project for first year undergraduate students in the field of Human-oriented Ambient Intelligence (shortly called Human Ambience), the domain of physical endurance training for cyclists is addressed. For long distance cyclists it is a challenge to train in an optimal fashion. The goal of training is to shift the basic critical power point upwards such that the person can deliver more power without having to pay back later (e.g., $[9,17])$. This can be accomplished by conducting training in intervals with a power level above the critical power point (high effort intervals), alternating with intervals with power level below the critical point (low effort intervals). The goal of the project is to design and use an ambient agent guiding the cyclists within training experiments that are to be set up. Equipment is used to measure and display the provided power and the heart rate, and the model presented in the current paper is used to estimate the dynamical critical power point on the fly. Based on this estimated value, per interval the ambient agent gives advice on the power to be provided (for low effort intervals below the critical power point, and for high effort intervals above it).

Conducting this project (scheduled in four weeks time) requires substantial effort from students, among which setting up an appropriate series of experiments and identifying appropriate parameter values in the model. The experiences overall are positive; the project as set up is a permanent subject in the Human Ambience curriculum. Experiences for the project in June 2009 as formulated by the four different student groups (of two or three persons each) are as follows:

- During the exercising it turned out that the model gave a rather accurate image of the energy stores of the cyclist. Each time that the cyclist was about to stop cycling due to fatigue, the model gave a warning that he could take it more easy. The cyclist noticed that everytime when he was close to exhaustion, the model indicated that he could slow down the cycling. So, in this respect the model seemed well-adapted to him.

- During the training the cyclist could accommodate well to the model. The training was not too difficult or too easy. It never occurred that the cyclist was so exhausted that he could not follow the desired provided power. The heart rate was never above the bound of 160 . The cyclist found training with the model a nice experience.

- The model took care that there was enough rest between the intervals, due to which the cyclist could not become exhausted and was cycling in the neighbourhood of the critical power point. In this manner it still was an intensive training.

- At the end of the training the cyclist was not really tired. He felt that effort had been spent, but still was able to spend extra effort. It turned out that according to the 
Fig. 4 Overview of the OFS-model

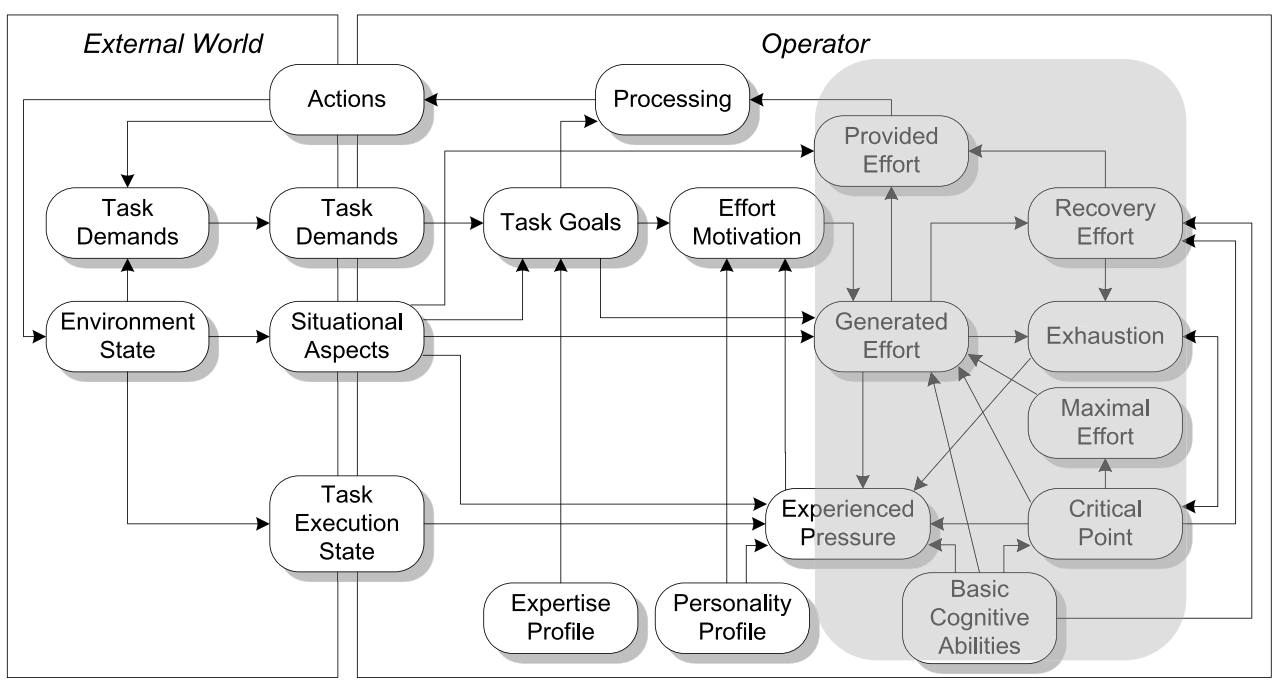

model the critical power point had decreased much more than was the case in reality.

Although the low number of persons does not justify a conclusive evaluation, from these evaluations at least it turned out that in three of the four groups the students were able to find settings for which they experienced realistic advices. In one of the four groups it was reported that they experienced that the model indicated too low effort. A crucial issue here is that the model contains a number of parameters, and most of the values of these parameter are personspecific, may also be affected by training. Estimating realistic values for these parameters for such a dynamical model is a real challenge, as almost no direct objective measurements are possible. What is shown in the reports above is a more subjective perspective, taking into account in how far the cyclist feels the level of fatigue the model assumes. But even when such a feedback is used, and deviations are noticed, due to the number of parameters it is far from trivial how to adjust values of parameters in such a manner that the results become better. Nevertheless, it seems that most of the student groups were able to find satisfactory settings. This issue of parameter estimation also plays a main role in the validation discussed in Sect. 6.2.

\subsection{Validation of the model in experiments with a functional state model}

The model presented in the current paper has been applied in a more cognitive task context as well. More specifically, it has been the inspiration for a part of the functional state model of an operator performing demanding tasks described in [1]. This operator function state (OFS) model, depicted in Fig. 4, also includes additional elements such as effort motivation and experienced work pressure. The shaded area is what corresponds to the model presented in the current paper.
As input the OFS model uses the external factors task demands and environment state. Moreover, it takes into account personal characteritics: experience, cognitive abilities and personality profile. Both are used to determine a person's (dynamical) state. The human's actions with respect to the task (e.g. performance quality), are represented in the Task Execution State. Exhaustion builds up or reduces over time according to the model described in the previous sections: when the generated effort is above the dynamic critical power point, exhaustion increases, otherwise exhaustion decreases, depending on the level of recovery effort. In addition an experienced pressure state is modelled. This is a more instantaneous state than exhaustion; the latter builds up over time more slowly. The temporal relation for experienced pressure is based on the previous experienced pressure and a change value based on the states connected to it. This model also determines the generated effort, as depending on a state of effort motivation, among others. This is not modelled in the model described in the previous sections; there generated power was assumed as depending on a personal choice of the person (free will), out of the scope of the model (external variable), although constraints are indicated by the dynamic maximal power $D M P$.

A first test of the OFS-model against empirical data in a simulation-based training environment ${ }^{3}$ has shown preliminary results which were positive; see [2]. In the validation study 31 persons participated (18 males, 13 females, of which 25 students). They ranged in age from 17 to 57 years with a mean age of 26 years. The experiment took approximately 1 hour for which participants received a voucher of 10 euro. In addition, there was a voucher of 100 euro for the one with the best score.

${ }^{3}$ For a demo of a version of this simulation-based training environment, see http://www.forcevisionlab.nl/demo/MissileCommandWebStarter. html. 
In the experiment the main task is a task where the goal is to get as many points as possible by eliminating hostile objects. Objects (friends and enemies) are falling down from different locations at different speeds. The purpose is to shoot the enemies before they hit the ground, and not shoot the friends. Shooting at a missile is done by a mouse click at a specific location; the missile would then explode exactly at the location of the mouse click. The speed with which the missile reaches this location is 79.6 pixels per second. When an object is within a radius of 50 pixels of the explosion, the object is destroyed. The number of points a participant receives for hitting an enemy is proportional to the proximity of the explosion. When a participant shoots a friend or when an enemy reaches the bottom of the screen, points are lost. When a friendly object reaches the bottom of the screen points are gained. Next to each of the objects, a calculation is written on the screen, representating an identification task. A correct calculation indicates that the object is friendly. An incorrect calculation indicates that the object is an enemy.

In [2] it is reported how automated parameter estimation for the functional state model was used two different methods: a gradient-based approach and a simulated annealing approach based on probabilistic search. For both cases the performance level of the operator in terms of numbers of enemies shot before landing and numbers of friendly objects left unaffected was used as an evaluation criterion.

To perform gradient-based parameter estimation, a method based on the maximum likelihood principle has been applied [16], based on a likelihood function of the measurement data and the unknown parameters. This function is essentially the probability density function of the measurement data given the parameter values. For more details, see [2]. The Simulated Annealing method used a probabilistic technique to find a parameter setting. In this method a random parameter setting is chosen as the best available parameter setting at the start. Then a displacement is introduced into these settings to generate a neighbour of the current parameter settings in the search space. If this neighbour is found to provide a more appropriate representation of the observed human behavior, then it is marked as the best known parameter setting; otherwise a new neighbor is selected to evaluate its appropriateness. The displacement in the parameter settings depends on the temperature, in case the temperature is higher, the steps will become larger. Over time the temperature goes down, thus obtaining a form of convergence. For more details about this form of parameter estimation, see [2].

The results obtained for the parameter adaptation overall were satisfactory. However, a number of parameters $(35 \%$ in average) were evaluated as less accurate, and, therefore, less reliable. Partially this can be explained by a large overall number of 30 parameters being estimated. Most of the less precise parameters have a weak relation to the measured output (e.g., noise sensitivity). Furthermore, since the empirical data were collected based on irregular events (i.e., actions of humans), some intervals contained the amount of information insufficient for estimation. Despite this, as shown in [2], the models with estimated parameters demonstrated good predictive capabilities in the cross-validation, which is a strong indicator of the model validity.

\section{Discussion}

In this paper a virtual human agent model was introduced that addresses the notion of critical power which plays a central role in scientific and practical literature on exercising and sport. It performs integration of monitoring data on generated power over time and uses this to determine in a dynamic manner the critical power as an indicator for the amount of stored resources left. The model realises homeostasis under the assumption that the human uses this indicator to make the proper decisions about its generated power. Decision criteria for generated power are, for example, keeping this indicator constant (achieving a steady state), or keeping the indicator between certain bounds, thus avoiding running out of resources. It has also been discussed how a person's heart rate can be related to the dynamic critical power and used as an indicator. To keep the complexity of the model limited, the following assumptions were made.

- The extraction of power from the resources and recovery are without loss: all power invested in recovery adds to the stable power point, and all extraction subtracts from the stable power point. This implies that during a period for which the stable power point is the same at the start and at the end, the sum of all extracted power is equal to the sum of all invested recovery power.

- The internal decision mechanism for the cyclist is left out of consideration.

- More specific aspects such as oxygen uptake, food intake, and lactic acid production have been left out of consideration.

For each of these assumptions possible extensions of the model can be made to loosen them.

The model has been used to perform a number of simulations, one of which was presented above. Moreover, a formal analysis has been undertaken that shows under which conditions the critical power indicator indeed correlates to what it is expected to indicate, and that some strategies often used are guaranteed to work out well. A first test of the model against empirical data has shown preliminary results which were positive.

The agent model may be useful in a number of application areas for intelligent agents. In the first place it may by useful as a basis for virtual characters with a realistic appearance (which, for example, may show a heart rate depending 
on efforts made). Furthermore, the agent model may be applied as part of an intelligent ambient agent device interacting with humans in physical exercise or sport, or in other demanding circumstances. Supporting devices mostly they concentrate on the sensoring and do not possess much intelligence to perform analysis of sensor data. Incorporating a dynamical model may provide a basis for more intelligent ambient agents. As an example of this development, some of the ideas reported in the current paper already were the inspiration for a part of the functional state model described in [1]. Moreover, the model may be used for social simulations, to investigate how persons can cooperate in order to manage their resources more economically. For example, in cycling sports, based on riding behind each other sophisticated cooperation strategies between competing teams are followed, including negotiation processes. Finally, more specifically for the cycling case, when also ascending and descending roads are taken into account, in addition to air resistance, also gravitation resistance can be built in. Gravitation resistance is independent of velocity, but depends on the steepness $s$ of the road (taken as the tangens $\tan (\alpha)$ with $\alpha$ the angle), and is proportional to the combined mass $m$ of the cyclist and the bike. An approximation for not too large $s$ is that for ascending roads gravitation resistance contributed to the total resistance is also proportional to $s$, with the gravitation constant $g$ as the proportion factor: gms (for larger $s$ the formula $g m s / \sqrt{ }\left(1+s^{2}\right)$ can be taken). For descending roads a negative contribution to the resistance (proportional to $m s / \sqrt{ }\left(1+s^{2}\right)$ with proportion factor $g$ ) can be taken into account. In principle this can lead to a situation where the gravitation alone is enough to compensate the air resistance, or even more. One of the questions that can be addressed is the following: suppose you decrease the mass $m$ by buying a more expensive, lighter bike (10 kg instead of $16 \mathrm{~kg}$ ), and by reducing your own weight (from 74 to $70 \mathrm{~kg}$ ), how much faster can you climb an ascending road of say $10 \%$ using the same power?

Acknowledgements The cycling endurance training experiments briefly reviewed in Sect. 6.1 were conducted by Fiemke Both, Bas van der Drift, Araik Ghazarian, Rolf Gigengack, Tineke van den Hoek, Rianne van Lambalgen, Roxane Lubbers, Ivar Meijs, Simon Provoost, Hugo Slinger, and Sascha Slisser. The author is grateful to the anonymous reviewers who proposed substantial improvements in the text.

Open Access This article is distributed under the terms of the Creative Commons Attribution Noncommercial License which permits any noncommercial use, distribution, and reproduction in any medium, provided the original author(s) and source are credited.

\section{Appendix: Some further details of the formal analysis}

This Appendix gives some more details such as lemma and proof sketches of this lemma and the propositions.
Maintaining a steady state Enduration sporters often try to maintain what they call a steady state: a state in which the main parameters are kept constant. In particular, it can be analysed how $D C P(t)$ can be kept constant. From the differential equation for $D C P(t)$ it follows:

$$
\begin{aligned}
d D C P(t) / d t= & \gamma_{1} \beta G P(t) \operatorname{Pos}(D C P(t)-G P(t)) \\
& -\gamma_{2} \operatorname{Pos}(G P(t)-D C P(t))
\end{aligned}
$$

Since never both $\operatorname{Pos}(x)$ and $\operatorname{Pos}(-x)$ are nonzero, $d D C P(t) / d t=0$ is equivalent to

$G P(t) \operatorname{Pos}(D C P(t)-G P(t))=0$ and

$\operatorname{Pos}(G P(t)-D C P(t))=0$

This is logically equivalent to either

$G P(t)=0 \quad$ and $\quad P o s(G P(t)-D C P(t))=0$

or

$\operatorname{Pos}(D C P(t)-G P(t))=0$ and

$\operatorname{Pos}(G P(t)-D C P(t))=0$

or

$(B C P-D C P(t)) / B C P=0$ and

$\operatorname{Pos}(G P(t)-D C P(t))=0$

which can be rewritten to either

$G P(t)=0 \quad$ and $\quad D C P(t) \geq 0$

or

$G P(t)=D C P(t)$

or

$D C P(t)=B C P \quad$ and $\quad G P(t) \leq D C P(t)$

This analysis is summarised in the following theorem expressing how a steady state for $D C P(t)$ can be characterised.

Theorem 1 (Maintaining a steady state) For any time point $t$, when $G P(t)>0$, the following are equivalent:

(i) $\frac{d D C P(t)}{d t}=0$ (dynamic critical power equilibrium)

(ii) either $G P(t)=D C P(t)$ or $G P(t) \leq D C P(t)=B C P$

How the indicator relates to the resources

A next issues to address in the analysis is the question how the dynamic critical power relates to the real resources. The level of resources $R(t)$ is assumed to be based on losses per 
time unit that are proportional to the extracted power (factor $\alpha_{2}$ ) and gains proportional to the recovery power (factor $\left.\alpha_{1}\right)$ :

$$
\frac{d R(t)}{d t}=\alpha_{1} R P(t)-\alpha_{2} E P(t)
$$

In a different form this can be expressed by.

$$
R\left(t_{2}\right)=R\left(t_{1}\right)+\alpha_{1} \int_{t 1}^{t 2} R P(t) d t-\alpha_{2} \int_{t 1}^{t 2} E P(t) d t
$$

Recall that by $B R$ the basic (additional) resources are denoted (in the literature often called $W^{\prime}$ ). When at time point $t_{0}$ the resources are the basic resources, then the model can be described as:

$R(t)=B R+\alpha_{1} \int_{t 0}^{t} R P(t) d t-\alpha_{2} \int_{t 0}^{t} E P(t) d t$

To analyse the relationships between the indicators and the resource level, two special extreme cases are considered first: the case that the values are equal to the basic values, as in a situation at rest (addressed in Proposition 1), and the case that the resource level is 0 , as in a situation with completely exhausted resources (addressed in Proposition 2). For proper functioning in both special cases certain conditions on the parameters are identified in the two propositions. These conditions also turn out sufficient for proper functioning for the general case, as covered by Theorem 2 .

In Proposition 1 it is investigated under which conditions the dynamic critical power is a proper indicator for the basic resources. It turns out that this is the case under a certain condition on the parameters; the same condition implies that the resource level is a linear function of the dynamic critical power. The proposition makes use of Lemma 1 which related the dynamic critical power to the resource levels.

Lemma 1 (Critical power vs resource level) For all time points $t_{1}$ and $t_{2}$ it holds:

$$
\begin{aligned}
\gamma_{1}\left(R\left(t_{2}\right)-R\left(t_{1}\right)\right)= & \alpha_{1}\left(D C P\left(t_{2}\right)-D C P\left(t_{1}\right)\right) \\
& +\left(\alpha_{1} \gamma_{2}-\gamma_{1} \alpha_{2}\right) \int_{t 1}^{t 2} E P(t) d t
\end{aligned}
$$

Proof of Lemma 1 Multiplying

$$
\begin{aligned}
& R\left(t_{2}\right)=R\left(t_{1}\right)+\alpha_{1} \int_{t 1}^{t 2} R P(t) d t-\alpha_{2} \int_{t 1}^{t 2} E P(t) d t \\
& D C P\left(t_{2}\right)=D C P\left(t_{1}\right)+\gamma_{1} \int_{t 1}^{t 2} R P(t) d t-\gamma_{2} \int_{t 1}^{t 2} E P(t) d t
\end{aligned}
$$

by $\gamma_{1}$ respectively $\alpha_{1}$ provides:

$$
\gamma_{1} R\left(t_{2}\right)=\gamma_{1} R\left(t_{1}\right)+\gamma_{1} \alpha_{1} \int_{t 1}^{t 2} R P(t) d t-\gamma_{1} \alpha_{2} \int_{t 1}^{t 2} E P(t) d t
$$

$$
\begin{aligned}
\alpha_{1} D C P\left(t_{2}\right)= & \alpha_{1} D C P\left(t_{1}\right)+\alpha_{1} \gamma_{1} \int_{t 1}^{t 2} R P(t) d t \\
& -\alpha_{1} \gamma_{2} \int_{t 1}^{t 2} E P(t) d t
\end{aligned}
$$

From subtracting, the statement of Lemma 1 follows.

Proposition 1 (Indicating basic resource levels) The following are equivalent:

(i) The dynamic critical power is a proper indicator for basic resources:

$$
\forall t[D C P(t)=B C P \Leftrightarrow R(t)=B R]
$$

(ii) $\gamma_{1} / \alpha_{1}=\gamma_{2} / \alpha_{2}$

(iii) For all time points $t_{1}$ and $t_{2}$ it holds:

$$
\alpha_{1}\left(D C P\left(t_{2}\right)-D C P\left(t_{1}\right)\right)=\gamma_{1}\left(R\left(t_{2}\right)-R\left(t_{1}\right)\right)
$$

(iv) The expression $\gamma_{1} R(t)-\alpha_{1} D C P(t)$ is invariant over time: for all time points $t_{1}$ and $t_{2}$ it holds:

$$
\gamma_{1} R\left(t_{2}\right)-\alpha_{1} D C P\left(t_{2}\right)=\gamma_{1} R\left(t_{1}\right)-\alpha_{1} D C P\left(t_{1}\right)
$$

(v) The dynamic critical power is a linear function of the resource level: for a given point $t_{0}$, for all time points $t$ it holds:

$$
\alpha_{1} D C P(t)=\gamma_{1} R(t)+\alpha_{1} D C P\left(t_{0}\right)-\gamma_{1} R\left(t_{0}\right)
$$

Proof of Proposition 1 (i) $\Rightarrow$ (ii) Suppose for two time points $t_{1}$ and $t_{2}$ it holds:

$R\left(t_{2}\right)=R\left(t_{1}\right)=B R \quad$ and $\quad D C P\left(t_{2}\right)=D C P\left(t_{1}\right)=B C P$

whereas between these time points resources have been extracted, after which full recovery took place, i.e.,

$\int_{t 1}^{t 2} E P(t) d t \neq 0$

By applying Lemma 1 it follows that $\alpha_{1} \gamma_{2}-\gamma_{1} \alpha_{2}=0$.

(ii) $\Rightarrow$ (i) This follows from Lemma 1, applied to the time interval between initial time point $t_{0}$ and $t$.

(ii) $\Leftrightarrow$ (iii) $\Leftrightarrow$ (iv) $\Leftrightarrow$ (v) This also follows from Lemma 1.

In Proposition 2 it is investigated under which conditions the dynamic critical power is a proper indicator for running out of resources. This is the case under a certain further condition on the parameters; this condition implies that the resource level is proportional to the dynamic critical power, as expressed in Theorem 2. Below it is assumed that at the initial time point $t_{0}$ the resources $B R\left(t_{0}\right)$ are the basic resources $B R$ and the dynamic critical power $D C P\left(t_{0}\right)$ is the basic critical power $B C P$. 
Proposition 2 (Indicating running out of resources) Suppose $\gamma_{1} / \alpha_{1}=\gamma_{2} / \alpha_{2}$ and let $\eta=B C P / B R$. Then the following are equivalent:

(i) The dynamic critical power is a proper indicator for running out of resources:

$\forall t \quad[D C P(t)=L C P \Leftrightarrow R(t)=0]$

(ii) At any point of time the dynamic critical power as an indicator faithfully (proportionally) reflects the resources left:

\section{$(D C P(t)-L C P) / R(t) \quad$ is constant over time}

(iii) $\gamma_{1} / \alpha_{1}=\gamma_{2} / \alpha_{2}=\eta$

Proof of Proposition 2 (i) $\Rightarrow$ (iii) Apply Proposition 1(iii) to a time interval from the initial time point $t_{0}$ and a time point $t$, to obtain that the expression $\alpha_{1} D C P(t)-\gamma_{1} R(t)$ is invariant over time. From this it follows that for all $t$

$\alpha_{1} D C P(t)-\gamma_{1} R(t)=c$

with

$c=\alpha_{1} D C P\left(t_{0}\right)-\gamma_{1} R\left(t_{0}\right)=\alpha_{1} B C P-\gamma_{1} B R$

From this it follows that

$$
\begin{aligned}
& {[\forall t \quad D C P(t)=L C P \Leftrightarrow R(t)=0]} \\
& \quad \Leftrightarrow \quad c=\alpha_{1} L C P \\
& \quad \Leftrightarrow \quad \alpha_{1}(B C P-L C P)=\gamma_{1} B R \\
& \quad \Leftrightarrow \quad \gamma_{1} / \alpha_{1}=\eta \\
& \quad \Leftrightarrow \quad \forall t \quad D C P(t)-L C P=\eta R(t)
\end{aligned}
$$

So under these conditions the dynamic critical power $L C P$ and the resource level are proportional over time with factor $\gamma_{1} / \alpha_{1}=\gamma_{2} / \alpha_{2}=\eta$. This proves Proposition 2 .

The corollary follows from the differential equation

$$
\frac{d R(t)}{d t}=\alpha_{1} R P(t)-\alpha_{2} E P(t)
$$

for $R(t)$ by expressing $E P(t)$ and $R P(t)$ in $G P(t)$ and $D C P(t)$ and then, according to Theorem 2, replacing $D C P(t)$ by $L C P+\eta R(t)$. An alternative derivation is by taking the differential equation for $D C P(t)$ and replacing $D C P(t)$ by $L C P+\eta R(t)$.

Notice that the parameters $\alpha_{1}, \alpha_{2}$ are physiological parameters related to mechanisms for extraction from and recovery of resources. In contrast the parameters $\gamma_{1}, \gamma_{2}$ are neural or cognitive parameters, assumed to be set by the brain. To obtain a faithful indicator system, the relationships between the physiological and neural parameters as expressed in Theorem 2 are needed. It is assumed that within the brain the neural parameters stand in these relationships to the physiological parameters.

\section{References}

1. Bosse T, Both F, van Lambalgen R, Treur J (2008) An agent model for a human's functional state and performance. In: Jain L, Gini M, Faltings BB, Terano T, Zhang C, Cercone N, Cao L (eds) Proceedings of the 8th IEEE/WIC/ACM international conference on intelligent agent technology, IAT'08. IEEE Comput Soc Press, Los Alamitos, pp 302-307

2. Both F, Hoogendoorn M, Jaffry SW, van Lambalgen R, Oorburg R, Sharpanskykh A, Treur J, de Vos M (2009) Adaptation and validation of an agent model of functional state and performance for individuals. In: Yang J-J, Yokoo M, Ito T, Jin Z, Scerri P (eds) Proceedings of the 12th international conference on principles of practice in multi-agent systems, PRIMA'09. Lecture notes in artificial intelligence, vol. 5925. Springer, Berlin, pp 595-607

3. Cannon WB (1932) The wisdom of the body. Norton, New York

4. Clark A (1997) Being there: putting brain body and world together again. MIT Press, Cambridge

5. Coats EM, Rossiter HB, Day JR, Miura A, Fukuba Y, Whipp BJ (2003) Intensity-dependent tolerance to exercise after attaining $\mathrm{VO}_{2 \max }$ in humans. J Appl Physiol 95:483-490

6. Damasio A (1999) The feeling of what happens: body, emotion and the making of consciousness. MIT Press, Cambridge

7. Fukuba Y, Whipp BJ (1999) A metabolic limit on the ability to make up for lost time in endurance events. J Appl Physiol 87:853861

8. Fukuba Y, Miura A, Endo M, Kan A, Yanagawa K, Whipp BJ (2003) The curvature constant parameter of the power-duration curve for varied-power exercise. Med Sci Sports Exerc 35:14131418

9. Henritze J, Weltman A, Schurrer RL, Barlow K (1985) Effects of training at and above the lactate threshold on the lactate threshold and maximal oxygen uptake. Eur J Appl Physiol Occup Physiol $54: 84-88$

10. Hill AV, Long CNV, Lupton H (1924) Muscular exercise, lactic acid, and the supply and utilisation of oxygen. Proc R Soc Bri 97(I-III):438-475

11. Hill AV, Long CNV, Lupton H (1924) Muscular exercise, lactic acid, and the supply and utilisation of oxygen. Proc R Soc Bri 97(VII-VIII):155-176

12. Hill DW (1993) The critical power concept. Sports Med 16:237254

13. Jones AM, Wilkerson DP, DiMenna F, Fulford J, Poole DC (2008) Muscle metabolic responses to exercise above and below the "critical power' assessed using 31P-MRS. Am J Physiol Regul Integr Comp Physiol 294:585-593

14. Lambert EV, St Clair Gibson A, Noakes TD (2005) Complex systems model of fatigue: integrative homoeostatic control of peripheral physiological systems during exercise in humans. Br J Sports Med 39:52-62

15. Noakes TD (2000) Physiological models to understand exercise fatigue and the adaptations that predict or enhance athletic performance. Scand J Med Sci Sports 10:123-145

16. Sorenson HW (1980) Parameter estimation: principles and problems. Dekker, New York

17. Stasiulis A, Ančlauskas R, Jaščanin J (2000) The effects of training intensity on blood lactate breakpoints in runners. J Hum Kinet 3:17-26 
18. Steels L, Brooks R (1995) The artificial life route to artificial intelligence: building embodied, situated agents. Erlbaum, Hillsdale

19. Wilmore JH, Costill DL (2004) Physiology of sport and exercise. Human Kinetics, Champaign

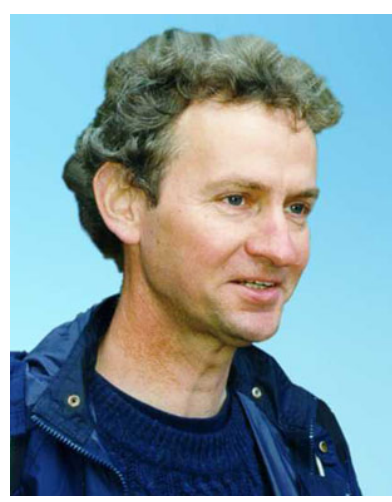

Jan Treur has a full professorship in Artificial Intelligence at Vrije Universiteit Amsterdam (VUA) since 1990. He is heading the department of Artificial Intelligence consisting of about 45 researchers. He is an internationally well-recognized expert in agent technology, cognitive modelling and knowledge engineering. He is or has been member of the programme committee of many of the main conferences and workshops and many journals in these areas. His extensive list of publications (e.g., see www.cs.vu.nl/ treur) covers major scientific publication media in artificial intelligence and cognitive science, including the top level conferences and journals. Some of his recent involvements are organising and chairing the series of international workshops on Human Aspects in Ambient Intelligence (HAI) at the European Conference on Ambient Intelligence (AmI'07), at the International Conference on Intelligent Agent Technology, IAT'08 and IAT'09. Moreover he initiated and designed the strongly multidiciplinary Bachelor study program Human Ambience at VUA in Human Ambience, combining subjects from Artificial Intelligence, Computer Science, Psychology and Biomedical Sciences, which forms a main stream in the study Lifestyle Informatics. 